\title{
Evaluation of Epithelioid Trophoblastic Tumor in the Light of Literature
}

\author{
Sefa Kurt, $\mathrm{MD}^{1}$; Orkun IIgen, $\mathrm{MD}^{1 *}$; Emine Cagnur Ulukus, $\mathrm{MD}^{1}$; Resmiye Irmak Yuzuguldu, MD²; Murat Celiloglu, MD ${ }^{1}$ \\ 'Department of Obstetrics and Gynecology, Dokuz Eylul University School of Medicine, Izmir, Turkey \\ ${ }^{2}$ Department of Pathology, Dokuz Eylul University School of Medicine, Izmir, Turkey
}

\begin{abstract}
Epithelioid trophoblastic tumors (ETTs) are extremely rare gestational trophoblastic neoplasia and a subtype of the placental site trophoblastic tumors (PSTTs). To our knowledge, there have been only 110 patients diagnosed with the ETT. ETT is generally seen in the reproductive period, following term pregnancy. Generally, as in PSTT, $\beta$-HCG levels are normal or slightly elevated. The most common complaint is abnormal vaginal bleeding. At the time of diagnosis, findings of metastasis can be seen in $50 \%$ of the cases. Transvaginal ultrasonography (TV-USG) and computed tomography (CT) are used for imaging in the literature. Surgical treatment and follow-up are sufficient in the early stages. We present a case of a 37-year-old ETT patient who suffered from irregular vaginal bleeding.

Keywords: Epithelioid trophoblastic tumor, Gestational trophoblastic neoplasia, Placental site trophoblastic tumor

Cite this article as: Kurt S, Ilgen O, Ulukus EC, Yuzuguldu RI, Celıloglu M. Evaluation of epithelioid trophoblastic tumor in the light of literature. Arch Iran Med. 2021;24(7):579-582. doi: 10.34172/aim.2021.81
\end{abstract}

Received: January 9, 2021, Accepted: May 5, 2021, ePublished: July 1, 2021

\section{Introduction}

Epithelioid trophoblastic tumors (ETTs) are extremely rare gestational trophoblastic neoplasia and a subtype of the placental site trophoblastic tumors (PSTTs). ETTs, first described by Shih et al in 1998, are rare gestational trophoblastic neoplasms that develop from the middle trophoblastic cells of the chorion laeve. ${ }^{1}$ There are 110 cases reported as of $2019 .{ }^{2}$ ETTs are similar to PSTT in terms of clinical behavior, pathological, and prognostic features. They are generally seen in the reproductive period and following a term pregnancy, and less frequently following abortion and molar pregnancy. It is reported that the average time from the last pregnancy to diagnosis is 76 months (2-300 months). ${ }^{2,3}$ The most common presentation at the time of diagnosis is irregular vaginal bleeding. This is followed by nonspecific complaints such as amenorrhea, abdominal pain, and abdominal bloating. $\beta$-HCG ranges widely from normal to slightly high. ${ }^{4}$ Because of the nonspecific initial complaints and slow tumor progression, diagnosis is delayed in ETTs, and findings related to metastasis or metastasis are observed in nearly $50 \%$ of the cases at the time of diagnosis. ${ }^{2-4}$

We present a case of a 37-year-old ETT patient who referred to our clinic due to irregular vaginal bleeding.

\section{Case Report}

A 37-year old female who referred to Dokuz Eylul University Hospital with abnormal bleeding had two children. The last delivery was 5 years ago via the vaginal route. She had no history of abortion or dilation and curettage $(\mathrm{D} \& \mathrm{C})$. The irregular vaginal bleeding started
3-4 years after birth and became more severe in the last months. On systemic examination of the patient, she was conscious, oriented, and cooperative. Vital signs and systemic examination findings were normal, except for mild pallor of the skin.

The uterus was 7-weeks pregnant size and mild vaginal bleeding was observed on gynecological examination. No other remarkable finding was found on gynecological examination. On transvaginal ultrasonography (TVUSG), the uterus measured $82 \times 67 \times 43 \mathrm{~mm}$. The endometrium was $10 \mathrm{~mm}$ and irregular. A heterogeneous mass was found in the corpus-isthmus region of the uterus with a diameter of $62 \times 54 \mathrm{~mm}$, containing hyperechogenic areas. Central blood flow was detected by Doppler USG.

The laboratory findings included mild anemia $(\mathrm{Hb}$ $10.5 \mathrm{~g} / \mathrm{dL}, \mathrm{Htc} 31.5)$. $\beta$-HCG value was measured at 17.94 $\mathrm{mIU} / \mathrm{mL}$. Thyroid function tests and coagulation tests were found to be normal. ETT was considered a result of the endometrial histopathological examination of the patient who underwent D\&C.

Computed tomography (CT) was used to scan the body for distant metastasis. No sign of distant metastasis was detected. The uterus was in the shape of an hourglass, and a marked enlargement at the level of the isthmus towards the cervix was observed intraoperatively (Figure 1). There was no sign of extrauterine spread in the pelvis. Bilateral ovaries, fallopian tubes, other pelvic organs, and upper abdominal organs were observed with normal appearance and structure. Following the intake of peritoneal irrigation fluid, hysterectomy and bilateral salpingectomy were performed while protecting the ovaries. When the 


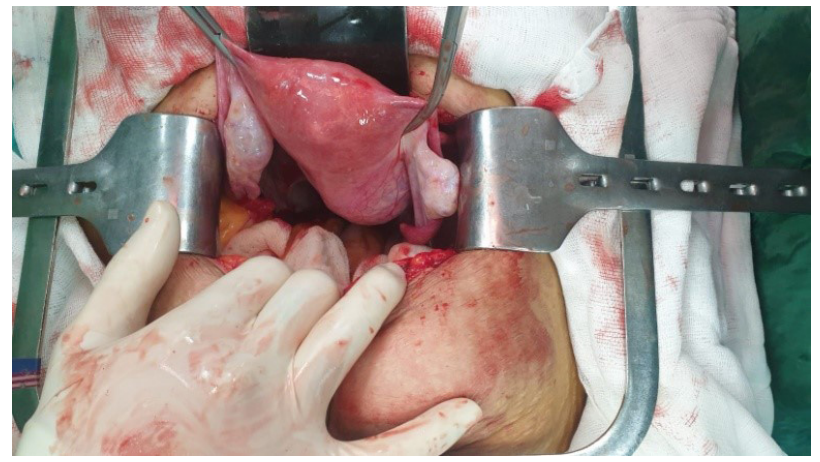

Figure 1. Intraoperative Image of the Tumor at the Level of the Isthmus-Corpus Region.

uterus was opened in the operation room, a necrotic, solid tumoral area with bleeding, a distinct demarcation line with the surrounding myometrium at the level of the corpus-isthmus region, was observed (Figure 2).

$\beta$-HCG levels decreased to the normal range after the 3rd day of the surgery and the patient was discharged in good conditions. The final pathology result was reported compatible with ETT (Figure 3). The patient came in for control in the postoperative $3 \mathrm{rd}$ month. No pathology was detected on the 3rd-month positron emission tomography - CT (PET-CT) of the patient.

\section{Discussion}

ETT is a rare trophoblastic neoplasia. One hundred and ten cases have been reported until 2019. ${ }^{2}$ ETTs are similar to PSTTs in terms of clinical, pathological, and prognostic features. Postmenopausal cases are rarely reported. It is reported that they are generally seen in the reproductive period, followed by term pregnancy, and less frequently after molar hydatidiform and abortion. ${ }^{5} \beta$-HCG levels are generally normal or slightly elevated in ETT as in PSTT. In our case, the serum $\beta$-hCG level was $17.49 \mathrm{mU} / \mathrm{mL}$ at the time of diagnosis. It has been reported in the literature that serum $\beta$-hCG levels in ETTs frequently vary between 12 and $148.460 \mathrm{mIU} / \mathrm{mL}$ and remain below $2500 \mathrm{mU} /$ $\mathrm{mL}{ }^{4,6}$

In a review conducted by Palmar et al on 52 cases, they reported that the most common complaint at presentation was abnormal vaginal bleeding and the mean age of onset of the disease was $38 .{ }^{4}$ In our case, the reason for admission to our clinic was complaint of irregular vaginal bleeding. The last pregnancy of the 37-year-old patient had terminated approximately 60 months ago with a term vaginal delivery.

ETTs are generally diagnosed late due to their slow growth, nonspecific symptoms, and low $\beta$-HCG production. The average diagnosis period after the last pregnancy is reported at 76 months (2-300 months). ${ }^{2-4}$ Metastasis findings can be seen in one-third to $50 \%$ of the cases at the time of diagnosis. ${ }^{4-6}$ Our case was diagnosed approximately 60 months after the last birth, earlier than the average period reported in the literature. ETTs typically manifest as a separate, hemorrhagic, solid, and cystic lesion located in the fundus, lower uterine segment, or endocervix. They can mimic degenerated myoma on imaging. ${ }^{7}$ Furthermore, ETTs with cervical extension should be considered in the differential diagnosis of cervical cancers. ${ }^{3,8,9}$

Some diagnostic features are specified on USG, depending on the location and structural features of ETTs. A well-circumscribed hypoechoic halo surrounding the tumor on the grayscale has been shown in an ETT series of 12 cases. ${ }^{10}$ It has been suggested that the hypoechogenic halo that limits the tumor may arise from the interface formed by the enlarged lymph and blood vessels between the tumor and the surrounding tissue. ${ }^{8,10}$ We identified

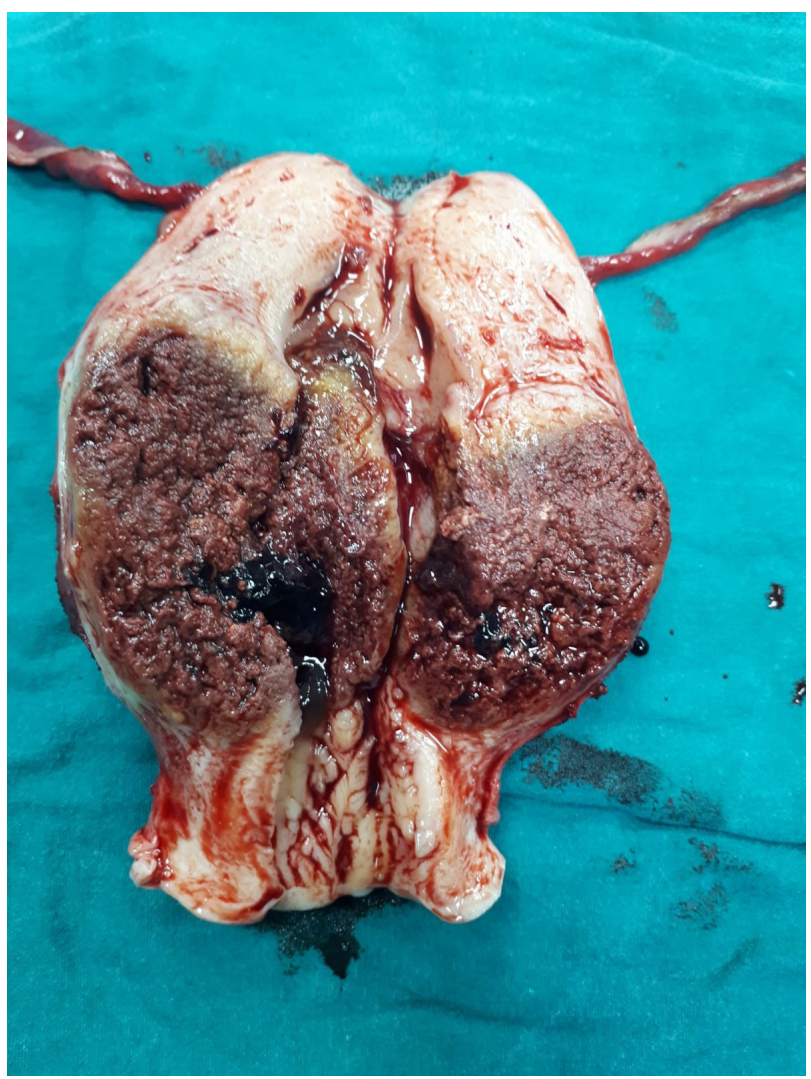

Figure 2. Macroscopic View of the Tumor.

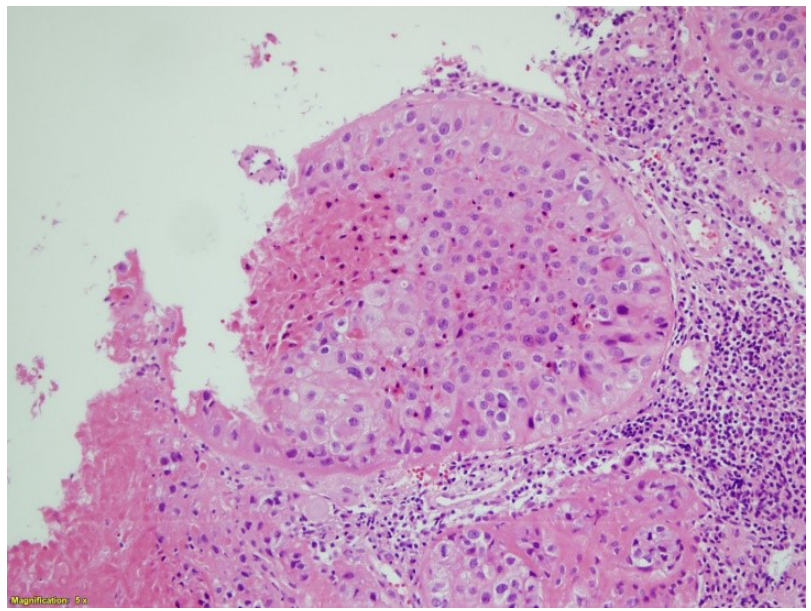

Figure 3. Microscopic View of the Tumor (H\&E stain, magnification power: 20x). 
this hypoechoic halo that limits the tumor on the TV-USG of our case. In addition, neovascularization and moderate central blood flow were detected in the tumor tissue on Doppler USG. It is reported that magnetic resonance imaging (MRI) and PET CT are useful in addition to USG and CT when determining the prevalence of ETT. ${ }^{6-8,11}$

Due to the limited number of cases, information about the prognosis of ETT is insufficient. ${ }^{2}$ Although Davis et al reported that the time elapsed since the previous pregnancy longer than 48 months is associated with a poor prognosis, Zhang et al did not confirm this finding., ${ }^{6,12}$ Current data show that the most important prognostic factor is the FIGO stage of the disease.,13

Our case was FIGO stage I. Therefore, this patient was treated with total abdominal hysterectomy plus bilateral salpingectomy. The ovaries were protected due to the young age of the patient. In the literature, adjuvant chemotherapy is recommended following debulking surgery in extensive disease; however, surgical treatment and follow-up are sufficient in early stages. ${ }^{3,14}$

ETTs are rare tumors. According to the data from the limited number of cases and limited series reported in the literature, it is understood that their diagnosis and treatment approach are similar to PSTTs.

In conclusion, evaluation and early diagnosis of these cases require adopting a broad perspective and a skeptical approach on clinical examination. Reporting the cases diagnosed each year and increasing the number of reported cases will enable us to have more information and experience about the diagnostic, treatment and prognostic features of the disease. Future advances in the management of patients with these rare diseases will enable us to better understand the biology of PSTT and ETT.

\section{Established Facts}

- Epithelioid trophoblastic tumor is an extremely rare tumor worldwide with 110 known cases reported.

- Prognosis and treatment protocols are not definite due to the small number of cases.

\section{Novel Insights}

Epithelioid trophoblastic tumors can be diagnosed by suspicion and adopting a broad perspective. They are best treated with a multidisciplinary team including gynecologic oncologists and pathologists.

\section{Authors' Contribution}

SK: manuscript writing, data collection; OI: review of the manuscript, data collection; ECU: review of the manuscript; RIY: manuscript writing; MC: supervision.

\section{Conflict of Interest Disclosures}

The authors declare that they have no conflicts of interest.

\section{Ethical Statement}

This report followed guidelines to be HIPAA compliant and permission was obtained from the patient to publish identifiable photographs. The study adhered to the tenets of the Declaration of Helsinki.

\section{Informed Consent}

Written informed consent was obtained from the patient for publication of this case report and any accompanying images.

\section{Funding}

None.

\section{References}

1. Shih IM, Mazur MT, Kurman RJ. Gestational trophoblastic disease and related lesions. In: Kurman RJ, TeLinde RW, eds. Blaustein's Pathology of the Female Genital Tract. 5th ed. New York: Springer-Verlag; 2002. p. 1193-250.

2. Gadducci A, Carinelli S, Guerrieri ME, Aletti GD. Placental site trophoblastic tumor and epithelioid trophoblastic tumor: clinical and pathological features, prognostic variables and treatment strategy. Gynecol Oncol. 2019;153(3):684-93. doi: 10.1016/j.ygyno.2019.03.011.

3. Horowitz NS, Goldstein DP, Berkowitz RS. Placental site trophoblastic tumors and epithelioid trophoblastic tumors: biology, natural history, and treatment modalities. Gynecol Oncol. 2017;144(1):208-14. doi: 10.1016/j. ygyno.2016.10.024.

4. Palmer JE, Macdonald M, Wells M, Hancock BW, Tidy JA. Epithelioid trophoblastic tumor: a review of the literature. J Reprod Med. 2008;53(7):465-75.

5. Keser SH, Kokten SC, Cakir C, Sensu S, Buyukbayrak EE, Karadayi N. Epithelioid trophoblastic tumor. Taiwan J Obstet Gynecol. 2015;54(5):621-4. doi: 10.1016/j. tjog.2015.08.020.

6. Zhang X, Lü W, Lü B. Epithelioid trophoblastic tumor: an outcome-based literature review of 78 reported cases. Int J Gynecol Cancer. 2013;23(7):1334-8. doi: 10.1097/ IGC.0b013e31829ea023.

7. Ohya A, Higuchi K, Shimojo H, Fujinaga Y, Kadoya M, Shiohara S. Epithelioid trophoblastic tumor of the uterus: a case report with radiologic-pathologic correlation. J Obstet Gynaecol Res. 2017;43(8):1360-5. doi: 10.1111/jog.13353.

8. Shaaban AM, Rezvani M, Haroun RR, Kennedy AM, Elsayes KM, Olpin JD, et al. Gestational trophoblastic disease: clinical and imaging features. Radiographics. 2017;37(2):681-700. doi: 10.1148/rg.2017160140.

9. McCarthy WA, Paquette C, Gundogan F, Lawrence WD. Comparison of p63 and p40 immunohistochemical stains to distinguish epithelioid trophoblastic tumor from other trophoblastic lesions. Int J Gynecol Pathol. 2018;37(4):4014. doi: 10.1097/pgp.0000000000000420.

10. Qin J, Ying W, Cheng X, Wu X, Lu B, Liang Y, et al. A wellcircumscribed border with peripheral Doppler signal in sonographic image distinguishes epithelioid trophoblastic tumor from other gestational trophoblastic neoplasms. PLoS One. 2014;9(11):e112618. doi: 10.1371/journal. pone.0112618.

11. Patel $\mathrm{T}$, Oldan J. Imaging of metastatic epithelioid trophoblastic tumor with 18F-FDG PET/CT. Clin Nucl Med. 2018;43(6):e200-e2. doi: 10.1097/rlu.0000000000002083.

12. Davis MR, Howitt BE, Quade BJ, Crum CP, Horowitz NS, Goldstein DP, et al. Epithelioid trophoblastic tumor: a single institution case series at the New England Trophoblastic 
Disease Center. Gynecol Oncol. 2015;137(3):456-61. doi: 10.1016/j.ygyno.2015.03.006.

13. Shen X, Xiang Y, Guo L, Ren T, Feng F, Wan X, et al. Analysis of clinicopathologic prognostic factors in 9 patients with epithelioid trophoblastic tumor. Int J Gynecol Cancer. 2011;21(6):1124-30. doi: 10.1097/IGC.0b013e31821dc89a.
14. Bolze PA, Attia J, Massardier J, Seckl MJ, Massuger L, van Trommel N, et al. Formalised consensus of the European Organisation for Treatment of Trophoblastic Diseases on management of gestational trophoblastic diseases. Eur J Cancer. 2015;51(13):1725-31. doi: 10.1016/j. ejca.2015.05.026.

(c) () 2021 The Author(s). This is an open-access article distributed under the terms of the Creative Commons Attribution License (http://creativecommons org/licenses/by/4.0), which permits unrestricted use, distribution, and reproduction in any medium, provided the original work is properly cited. 